\title{
AN ALGORITHM FOR BROADBAND NETWORK DIMENSIONING
}

\author{
Mette Røhne, Rima Venturin, Terje Jensen, Inge Svinnset \\ Telenor R\&D, P.O.Box 83, N-2027 Kjeller, NORWAY
}

\begin{abstract}
Dimensioning and designing broadband networks are big challenges for a network operator. Considering the functionality accompanying ATM-based networks, there is a necessity of having network planning procedures being able to exploit the advantages provided by ATM. One of the benefits is potential use of Virtual Paths, which then could compose a set of logical networks within the physical network. This paper presents algorithms for dimensioning broadband networks and for designing VP networks. The algorithms are based on a decomposition approach, where the locations are examined one by one. Therefore, more comprehensive relationships than found in most of the design algorithms using global optimisation could be applied. Due to the modularity further enhancements could be used within the algorithms with respect to, for instance, network element functionality, traffic handling principles, cost model, network design objectives and traffic approximations.

In this paper the algorithms for dimensioning and VP design are applied on two cases; in one case the variable rate traffic is represented by Statistical Bit Rate (SBR), while in the other case the variable rate traffic is defined as best-effort type of traffic and is characterised by Available Bit Rate (ABR). At the end, comparisons of some numerical results of these two cases are presented.
\end{abstract}

Keywords: Network design, dimensioning, VP design

\section{INTRODUCTION}

When designing broadband networks based on Asynchronous Transfer Mode (ATM) the potential gains achieved by handling traffic flows on Virtual Channel (VC) and Virtual Path (VP) levels should be considered (e.g. [1]). VP networks design is especially interesting because the use of VPs may result in many advantages, such as reduction in the processing and delay associated with the call acceptance control functions since the VPs are cross-connected in intermediate nodes. In addition, the use of VPs may simplify procedures in case of network failure, because having defined VPs faster reconfiguration could be obtained. On the other hand, employing VP networks could increase network transmission costs and decrease network throughput, because allocation of capacity to VPs restricts sharing of resources.

The VP network design may involve consideration of many other objectives, such as flexibility to changes in demand, network processing, set-up delay, end-to-end delay, 
set-up cost, switching cost, transmission cost, segregation of services, assurance of quality of service, reliability and throughput.

This paper presents algorithms for the broadband network dimensioning and costeffective VP design. The motivation for making these algorithms is described in Chapter 2. The algorithms described by main input/output data are covered in Chapter 3 . The same chapter contains the proposed cost model and short overview of traffic handling mechanisms. Some numerical results are given in Chapter 4 . The examples are used to illustrate the influence of the applications and service categories on the dimensioning and design of broadband networks. Applications using variable rate are characterised respectively by Statistical Bit Rate (SBR) and Available Bit Rate (ABR) and the resulting network solutions are compared. Finally, concluding remarks and plans for further work are given in Chapter 5.

\section{MOTIVATION AND SCOPE}

In ATM networks it is possible to have both switched VPs and VCs. The VPs are commonly handled as a part of the management plane, whereas VCs are normally handled as a part of the control plane. This categorisation implies that the VPs are changed over a longer timescale than the VCs. Therefore is the dimensioning of a VP logical network is medium-term, while each individual VC represents short-term planning.

A VP network, which consists of a set of VPs, may be seen as a logical overlay network, that may be compared to a physical network where the VPs correspond to the physical links and the switches terminating the VPs correspond to the nodes. On top of the physical infrastructure a number of VP networks can co-exist, sharing the same physical transmission and switching capacities. To illustrate how the use of VP networks affects the network performance two cases for VP networks can be looked at. In the first case the VP network is the same as the physical network, meaning that each physical link in the ATM network contains only one VP. The second case is a fully connected VP network, where every node will have one or several VPs to every other node. In the first case the network utilisation is maximised and the call blocking probability is minimised on a link. Advantage of the second case is that the processing and the cost at the intermediate nodes are at their minimal values. Therefore, the better design of VP networks will probably be somewhere between these two extreme cases depending on the cost for transmission capacity, set-up and switching.

VPs can be used for a number of reasons, like virtual networks for customers, segregating connections requesting different traffic characteristics and service quality requirements.

The challenge of designing networks can be approached in a number of ways ([1], [3], [6], [9]) During such activities, better network structures are sought where both the location of network elements and the topologies can be considered as variables.

\section{PROCEDURE FOR NETWORK DESIGN}

In the dimensioning procedure, presented in this paper, the locations of the network elements are given. The challenge is then to find the capacities of nodes and link sets under the constraints specified. Introducing the VP layer, the question of whether or not to cross-connect VPs should be raised. To investigate for an answer, trade-offs between possible savings in set-up/switching and the increase of other costs for having separate VPs should be compared. By introducing more VPs the link set capacities is divided 
into smaller units (optionally reducing the scale effect). This results typically in additional costs. Examining such trade-offs during the execution of the procedure, an objective function has to be defined. In principle, this function may differ for the different locations and could also differ from the way the network cost is calculated at the end. Compared with some other approaches (e.g. [5], [8]), the algorithm applied does not use a global optimisation formulation. Rather, the procedure has some resemblance with the decomposition approach in the sense that decisions with respect to traffic handling and capacities are made for each location in sequence, iteratively. A similar model has been applied in European projects $([2],[6])$.

\subsection{Overall procedure}

A general network design model is illustrated in Figure 1.

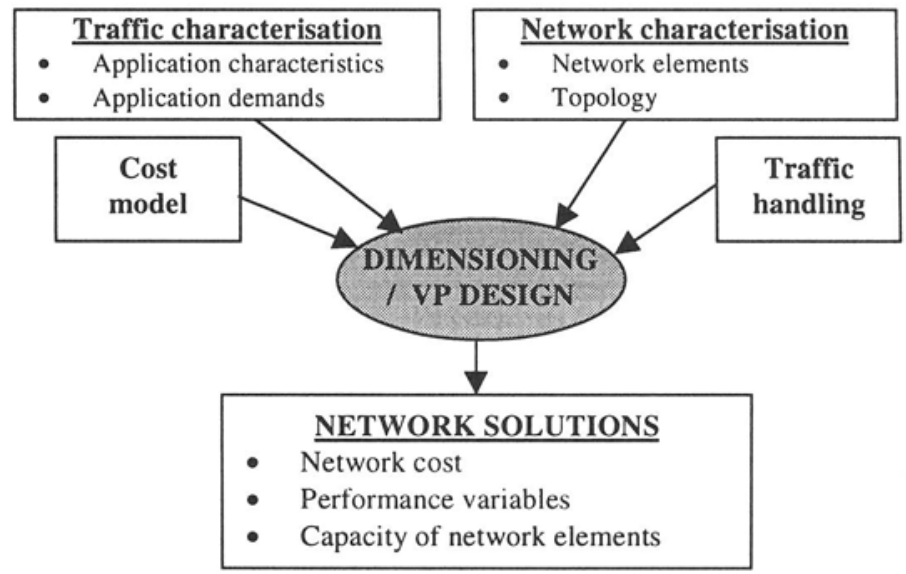

Figure 1. Input and output data for the dimensioning and VP design

The input data can be categorised as:

- Application characteristics. A number of bitstreams (connections) are associated with an application, potentially having different holding times and number of active periods during an invocation of an application. Each application is then described by a set of parameters, such as bitrates, real time requirements, acceptable cell loss ratios, and blocking probabilities, given for each bitstream associated with an application. Class of Service $(\mathrm{CoS})$ indicator can be used e.g. based on these parameters. Connections belonging to the same $\operatorname{CoS}$ are treated corresponding to the traffic handling schemes.

- Application demands are given by the arrival intensity. This measure is commonly referring to the time periods that are considered for dimensioning (reference periods).

- Characteristics of potential network elements. Information about interface modules, maximal capacities, and so forth, is specified for each of the network elements.

- Topology candidates like potential locations for nodes and link sets. 
- Traffic handling principles. As part of specifying traffic handling, routing rules and segregation/integration schemes are given. The routing rules are in this context given as specified alternate routes with the input data.

- Cost model. In order to find a cost-effective solution a model reflecting costs related to set-up, switching and transmission has to be described and applied in the algorithms.

\subsection{Traffic handling}

Traffic handling is the set of rules applied to manage the traffic in VPs and to handle establishment of connections. At the VP level, bitstreams with different characteristics can be segregated in a number of $\mathrm{CoS}$. Assigning $\mathrm{CoS}$ could be based on various criteria, such as bandwidth demands or Quality of Service (QoS) requirements. In [4], QoS classes are associated with different cell level QoS requirements (e.g. cell delay variation, cell loss probability). Using $\mathrm{CoS}$ is a possible way of giving a specified quality to particular bitstreams, e.g. by separating bitstreams into different VPs. As opposed to this, bitstreams can be integrated in VPs. In this case, the VP must support the QoS of the most demanding bitstream carried by that VP. To make integration of CoS cost-effective, proper buffer management schemes with time and/or loss priorities have to be introduced in the switching nodes. Bitstreams demanding high bandwidth may experience a severe accessibility performance. Therefore, they could be separated into different $\mathrm{CoS}$.

Requirements for set-up delay may have implications for routing and can be treated by introducing $\mathrm{CoS}$. Traffic handling when failure occurs can differ based on a number of requirements. For instance, different survivability classes could be incorporated in $\mathrm{CoS}$ assignment.

\subsection{Cost model}

In the procedure outlined, a cost model referring to a "local view" is requested. The "local view" approach applies that decisions are made sequentially for each node (one by one).

The cost factors used when executing the algorithm are identified as:

Transmission cost, $\mathrm{Zt}_{q}$; reflecting the cost of connections (bitstreams) between two nodes as well as termination units within those nodes, as shown in Figure 2. Considering two locations, $i$ and $j$, one transmission path from $i$ to $j$ with capacity $B_{i j}$, has a cost $C_{i j}$. A module for terminating such transmission paths in location $i$ has

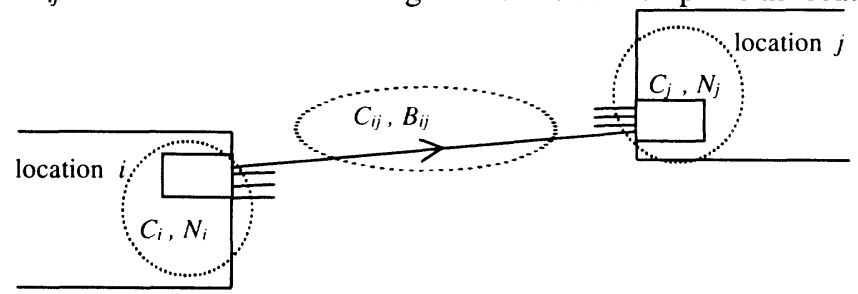

Figure 2. Illustrations of transmission cost factors 
a cost $C_{i}$ and may connect $N_{i}$ of these transmission paths (correspondingly for location $j$ ). Assuming a $V P_{q}$ having capacity demand $B_{q}$, the share of the bandwidth cost for that VP is calculated as:

$$
Z t_{q}=\frac{B_{q}}{B_{i j}} C_{i j}+\frac{B_{q}}{N_{i} B_{i j}} C_{i}+\frac{B_{q}}{N_{j} B_{i j}} C_{j}
$$

Switching cost, $\boldsymbol{Z s}_{q}$; reflecting the effort requested for transmitting ATM cells from an input port (incoming transmission path) to an output port (outgoing transmission path). This cost component is assumed to be proportional to the traffic load, $A_{b}$, and the equivalent bandwidth, $E B_{b}$, of the bitstream $b$. That is,

$$
Z s_{q}=\sum_{b \in q} Z s_{b}=\sum_{b \in q} A_{b}\left(\alpha \cdot E B_{b}+\beta\right)
$$

where $\alpha$ and $\beta$ are cost factors. The total cost component for a $V P_{q}$ is the sum of cost components of type $b$ carried by the corresponding VP.

Set-up cost, $Z c_{q}$; giving the processing requested for establishing (and releasing) a bitstream. Three types of processing steps could be incorporated as depicted Figure 3.

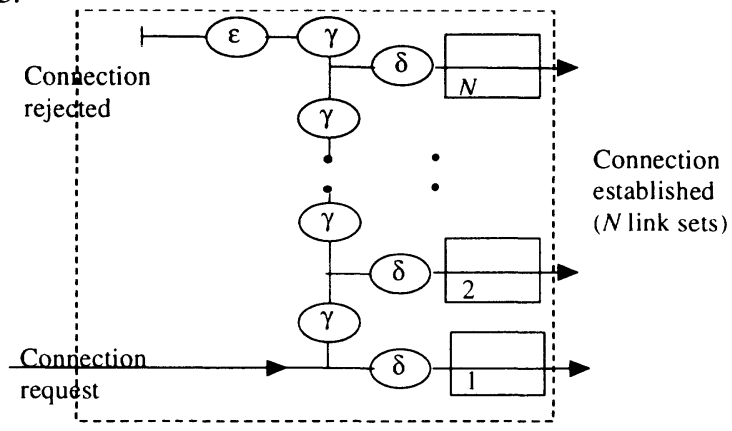

Figure 3 Illustrating the cost components for processing activities involved in connection set-up

Assuming there are $N$ link sets that could be used for establishing the connection, where link set $m$ as blocking probability $P b_{m}$, this cost component could be written as:

$$
\begin{gathered}
Z c_{q}=\sum_{b \in q} Z c_{b} \\
Z c_{b}=\frac{A_{b}}{T_{b}}\left\{\delta\left(1-P b_{1}\right)+\sum_{m=1}^{N-1}\left[(\delta+m \gamma) \prod_{k=1}^{m} P b_{k}\left(1-P b_{m+1}\right)\right]+(N \gamma+\varepsilon) \prod_{k=1}^{N} P b_{k}\right\}
\end{gathered}
$$

where bitstreams of type $b$ have a mean offered traffic $A_{b}$ with mean holding time $T_{b}$. The cost factors $\delta, \gamma$ and $\varepsilon$ express the effort related to a successful connection, an additional search for link sets and rejection of a connection request, respectively.

Considering the "local view" approach in the cost model described, different costs are compared and the solution resulting in the lowest cost is chosen. The contributions 
to the cost can be given weights. In this way the cost for a group of bitstreams, $Z_{Q}$, can be calculated as:

$$
Z_{Q}=\sum_{q \in Q}\left\{k_{t} Z t_{q}+k_{s} Z s_{q}+k_{c} Z c_{q}\right\}
$$

where $k_{t}, k_{s}$ and $k_{c}$ are relative weight factors related to transmission, switching and set-up cost. The relative weighting of the cost components is a difficult issue that may have significant implications on the logical network design.

It is therefore essential to perform sensitivity analyses varying the weight factors. In principle, these weight factors can be considered as taking part of the cost or placing relative credibility, e.g. when certain components are estimated with higher accuracy.

\subsection{Algorithm for network dimensioning and VP design}

The objective of the dimensioning procedure is to find the number of VPs that gives the highest reduction in the total network cost, including set-up, switching and transmission costs. The main outline of the dimensioning and VP design algorithms is illustrated in Figure 4. A fixed routing scheme is applied.

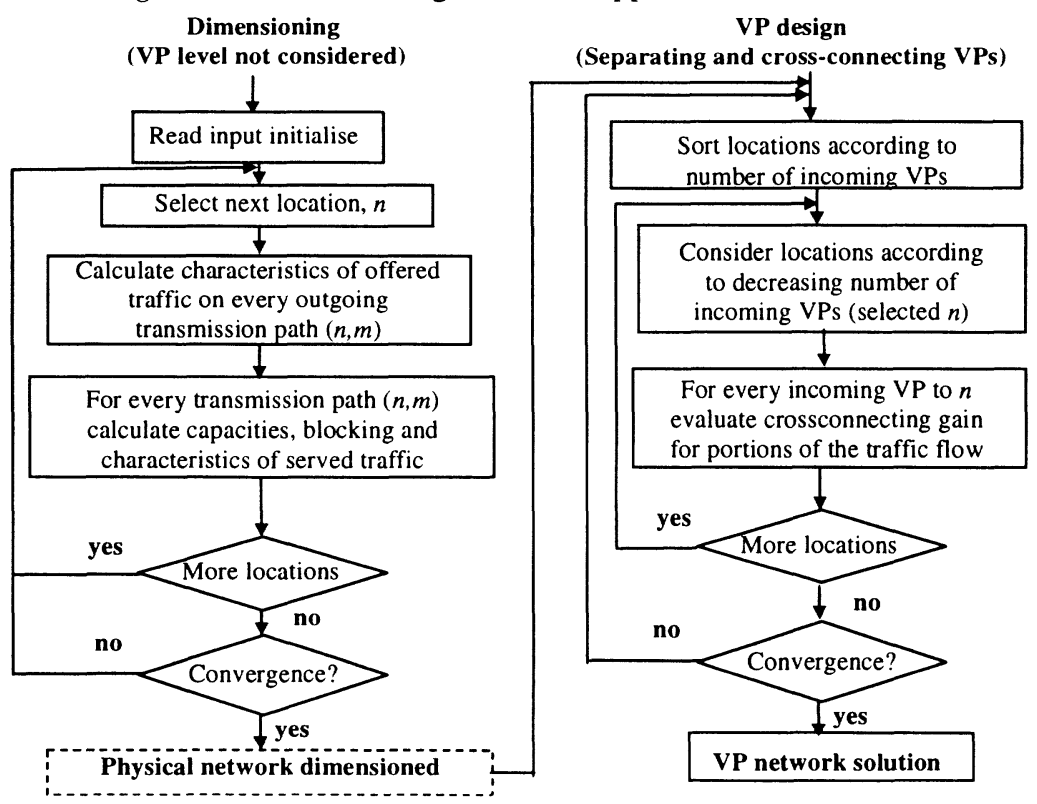

Figure 4. Outline of the broadband network dimensioning and VP design algorithm

In the first part the physical network is dimensioned without considering VPs and cross-connecting VPs. This implies switching each VC connection in every node it is crossing. In the calculations the "local view" approach has been used. The iteration is carried out until changes for the main variables (i.a. mean traffic and blocking probability) attached to the traffic streams are below the specified thresholds (convergence criteria). By separating and cross-connecting VPs the algorithm is looking 
for cheaper solutions. In the resulting network each physical link carries one VP with the capacity of the physical link set.

Before searching for VPs to be cross-connected the VP capacity is adjusted to the total demand whether there is segregation or not. The cost model and the relevant segregation scheme are used when deciding whether or not to cross-connect traffic streams. Traffic streams are segregated according to their $\mathrm{CoS}$ indicator.

Cross-connecting traffic streams means to separate the traffic streams from their previous VPs that terminate in the node and putting them into a new VP that is crossconnected in this node. This is accomplished by looking at one node at the time, starting with the node that has the highest number of incoming VPs.

VP capacity is a function of equivalent bandwidth and connection blocking probability. These are calculated based on the approximations described in [7]. Establishment/cross-connecting of VPs means a splitting of one VP into two where one of them is cross-connected through the node. The resulting sum of transmission bandwidth required for these two VPs will always be greater than or equal to the bandwidth of the VP that is split due to the scale effect. The extra bandwidth needed depends on the mixture of traffic contained in the VP to be split.

The costs, related to transmission, switching and set-up before and after the crossconnection, are calculated and compared. Splitting VPs increases the transmission cost. Cross-connecting a VP implies less switching and control activities, which leads to lower switching and set-up costs when the number of VPs increases. If the cost reduction is above a specified threshold, the traffic streams will be cross-connected in the node.

Applying these algorithms for the network dimensioning and VP design gives a cost-effective VP network solution.

\section{NUMERICAL EXAMPLES}

The algorithm outlined is applied on the example network depicted in Figure 5 to give some numerical examples. The network consists of 14 ATM switches, having both VC switching and VP cross-connecting.

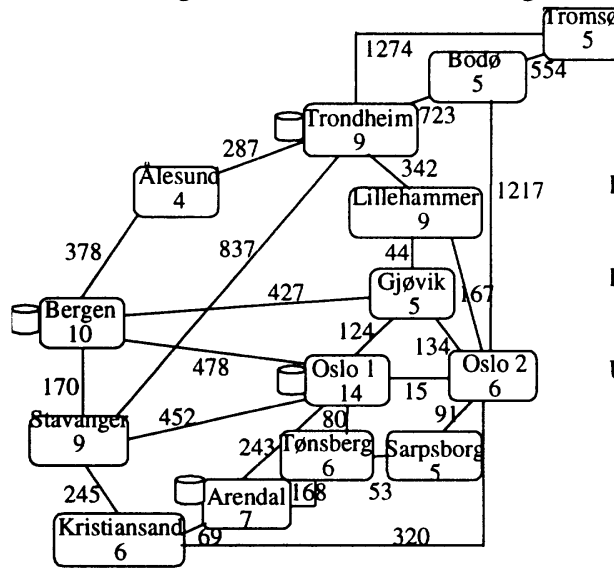

Lid - Location identity given by the name of the city where ATM node is located

Rd - Relative demand presented by a percentage of the total traffic generated

Uc - Unit cost per link between two locations

Servers for the Telegame application in Arendal, Oslo1, Bergen and Trondheim

Figure 5. Network structure, location identity, relative demand and unit cost 
Table I Applications with relevant input

\begin{tabular}{|c|c|c|c|c|c|c|c|c|}
\hline \multicolumn{9}{|c|}{ Application name } \\
\hline \begin{tabular}{|c|} 
Bit \\
stream \\
ID
\end{tabular} & \begin{tabular}{|c|} 
Direct \\
ion
\end{tabular} & ATC & $\begin{array}{l}\mathrm{MCR} \\
\text { /SCR } \\
\text { |kbit/s] }\end{array}$ & \begin{tabular}{|c|} 
PCR \\
$\mid$ |kbit/s| $\mid$
\end{tabular} & $P_{\text {lass }}$ & $\mathrm{P}_{\text {black }}$ & $\begin{array}{c}\mathrm{HT} \\
|\mathrm{min}|\end{array}$ & $\mathrm{CoS}$ \\
\hline \multicolumn{9}{|c|}{1 Telephony } \\
\hline 1.1 & UN & DBR & 64 & 64 & $10^{-6}$ & {$[0.01]$} & $\dddot{5}$ & i \\
\hline 1.2 & $\mathrm{NU}$ & $\mathrm{DBR}$ & 64 & 64 & $10^{-6}$ & 0.01 & 5 & $i$ \\
\hline \multicolumn{9}{|c|}{2 Video on Demand } \\
\hline 2.1 & UN & $\mathrm{DBR}$ & 8 & 8 & $10^{-9}$ & 0.01 & 90 & 2 \\
\hline 2.2 & $\mathrm{NU}$ & SBRR & 1664 & 2064 & $10^{-4}$ & 0.01 & 90 & 2 \\
\hline \multicolumn{9}{|c|}{3 Videoconference } \\
\hline 3.1 & $\mathrm{NU}$ & SBR & 100 & 2000 & $10^{-6}$ & 0.005 & 45 & 3. \\
\hline 3.2 & UN & SBR & 8 & 64 & $10^{-6}$ & 0.005 & 45 & 3 \\
\hline 3.3 & UN & DBR & 64 & 64 & $10^{-9}$ & 0.01 & 45 & 2 \\
\hline 3.4 & UN & $\mathrm{DBR}$ & 384 & 384 & $10^{-9}$ & 0.01 & 45 & 2 \\
\hline 3.5 & NU & DBR & 64 & 64. & $10^{-9}$ & 0.01 & 45 & 2 \\
\hline 3.6 & NU & DBR & 384 & 384 & $10^{29}$ & 0.01 & 45 & 2 \\
\hline \multicolumn{9}{|c|}{4 Real-time transaction } \\
\hline 4.1 & UN & SBR & 64 & 128 & $10^{-6}$ & 0.005 & 2 & 3 \\
\hline 4.2 & $\mathrm{NU}$ & SBR & 64 & 128 & $10^{-6}$ & 0.005 & 2 & 3 \\
\hline \multicolumn{9}{|c|}{5 Telegame } \\
\hline 5.1 & UN & DBR & 64 & 64 & $10^{-6}$ & 0.05 & 20 & 4 \\
\hline 5.2 & $\mathrm{NU}$ & SBR & 2000 & 5000 & $10^{-3}$ & 0.05 & 20 & 4 \\
\hline \multicolumn{9}{|c|}{$\begin{array}{l}\text { Direction: UN = user } \rightarrow \text { network, } \mathrm{NU}=\text { network } \rightarrow \text { user; } \\
\text { ATC = ATM Transfer Capability; } \mathrm{MCR}=\text { Minimum Cell } \\
\text { Rate; SCR = Sustainable Cell Rate; } \mathrm{PCR}=\text { Peak Cell Rate; } \\
\text { Ploss = Cell loss ratio requirement; Pblock = Connection } \\
\text { blocking requirement; } \mathrm{HT}=\text { Mean holding time } \text { CoS }=\text { Class } \\
\text { of Service, DBR = Deterministic Bit Rate. } \mathrm{SBR}=\text { Statistical } \\
\text { Bit Rate }\end{array}$} \\
\hline
\end{tabular}

Table 2 Total demand for each application

\begin{tabular}{|l|c|}
\hline Application name & $\begin{array}{c}\text { Total demand } \\
\text { [erlang] }\end{array}$ \\
\hline I Telephony & 151446 \\
\hdashline 2 Video on Demand & 2840 \\
\hline 3 Videoconference & 97567 \\
\hline 4 Real-time transaction & 48502 \\
\hline 5 Telegame & 998 \\
\hline
\end{tabular}

Table 3 Cost factors and weight factors for reference case

\begin{tabular}{|c|c|c|c|}
\hline Cost & Factor & Id & Value \\
\hline Transmission & weight & $k_{i}$ & 1.0 \\
\hline Switching & $\begin{array}{l}\text { weight } \\
\text { cost } \\
\text { cost }\end{array}$ & $\begin{array}{l}k_{x} \\
\alpha \\
\beta\end{array}$ & $\begin{array}{c}k \\
200.0 \\
0.0 \\
\end{array}$ \\
\hline Set-up & $\begin{array}{l}\text { weight } \\
\text { cost } \\
\text { cost } \\
\text { cost }\end{array}$ & $\begin{array}{l}k_{c} \\
\delta \\
\gamma \\
\varepsilon\end{array}$ & $\begin{array}{c}k \\
1.0 \\
1.0 \\
1.0 \\
\end{array}$ \\
\hline
\end{tabular}

Five applications are considered and are characterised in Table 1. The total demand per application is given in Table 2. In order to calculate elements of the traffic matrices, the total demand is multiplied by the relative demand for the source and destination location indicated in Figure $5^{1}$. Capacity of $155 \mathrm{Mbit} / \mathrm{s}$ is considered. The unit costs, $\mathrm{U}_{\mathrm{c}}$, are multiplied by 100 , in order to get the cost for having a single link between each pair of locations. One termination unit, able to handle one link, is assumed to have a cost equal to 10000 .

The values of the cost are given in Table 3. The VP capacity may be higher than a single link. For the calculations presented weight factor, $k$, is introduced and is equal to the weight factors for set-up and switching, $k=k_{c}=k_{s}$. As a minimum 50 VPs will be established, one for each direction on every link. The maximum number of VPs that may be established with the given network structure and with four $\operatorname{CoS}$ values is 448 .

In the reference case the configuration and the values are kept as presented in Figure 5 and in Table 1, 2 and 3. The numerical studies are made for two cases; in the first case the bitstreams with variable rate are characterised by SBR, while in the second by ABR.

\footnotetext{
${ }^{1}$ For example, the element in the traffic matrix for the Videoconference application as demand from Bodø to Bergen is found as: $97567 \cdot 5 / 100 \cdot 10 / 100$.
} 


\subsection{Reference case}

In this case variable rate bitstreams are characterised by SBR. For each SBR bitstream, the equivalent bandwidth has a value between the Sustainable Cell Rate (SCR) and Peak Cell Rate (PCR) dependent on the capacity of the VP.
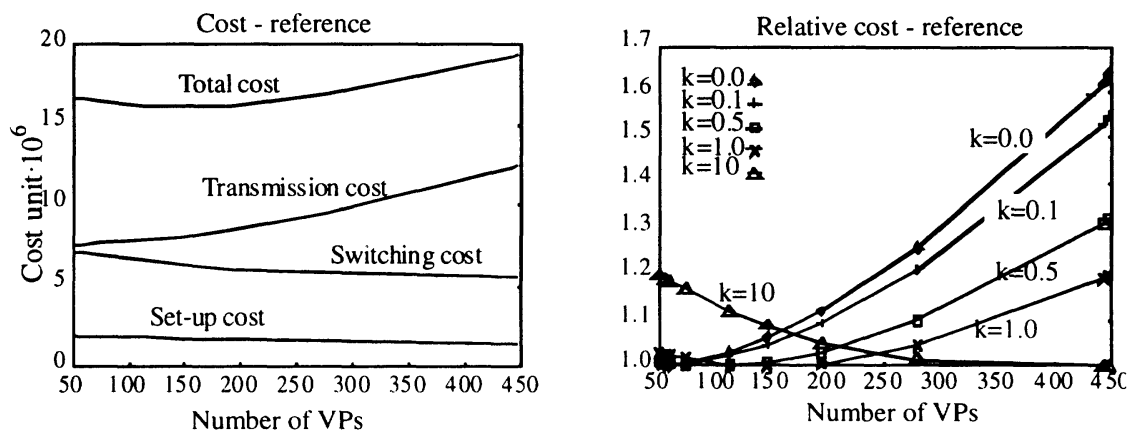

Figure 6. Results for reference case: a) Cost as function of number of VPs, b) Total cost relative minimum total cost for selected values of set-up/switching weight factors

Figure $6 a$ ) illustrates how the VP transmission cost is increasing as a function of the number of VPs.

As the costs related to set-up and switching are increasing, the establishment/crossconnection of VPs will become more profitable. A set of curves for the relative total cost for a selected number of set-up/switching weight factors, are depicted Figure $6 b$ ). The relative costs are found by dividing the total cost obtained by the minimum total cost for the relevant weight factor value $k$. The minimum total cost for the reference case, with the weight factor set to 1.0, is obtained when there are 146 VPs established.

As seen from the curves, a larger number of VPs are found as the better solutions (minimum relative cost) when greater weight is placed on set-up/switching cost. The shapes of the curves are explained by this effect. In one respect, these curves show the "goodness" of the solution found compared to alternative solutions. For instance, in case $k=1.0$, having 50 VPs gives a total cost that is ca. $3.0 \%$ more expensive than the better solution having 146 VPs, while the total cost having 448 VPs gives an increase of $19.9 \%$.

\subsection{ABR case}

In this case, the bitstreams with variable rate is characterised by $A B R$. The dimensioning algorithm is using the MCR, having the same value as the SCR in the reference case, as the equivalent bandwidth.

Similar results as for the reference case is depicted in Figure 7. The overall network cost is reduced since the equivalent bandwidth used is significantly lower, resulting in less bandwidth needed and less switching cost since the switching cost also is influenced by the equivalent bandwidth. The equivalent bandwidth of $\mathrm{ABR}$ is constant and therefore independent of the VP capacity. The capacity when splitting VPs is only increased in order to satisfy the required connection blocking probability. 

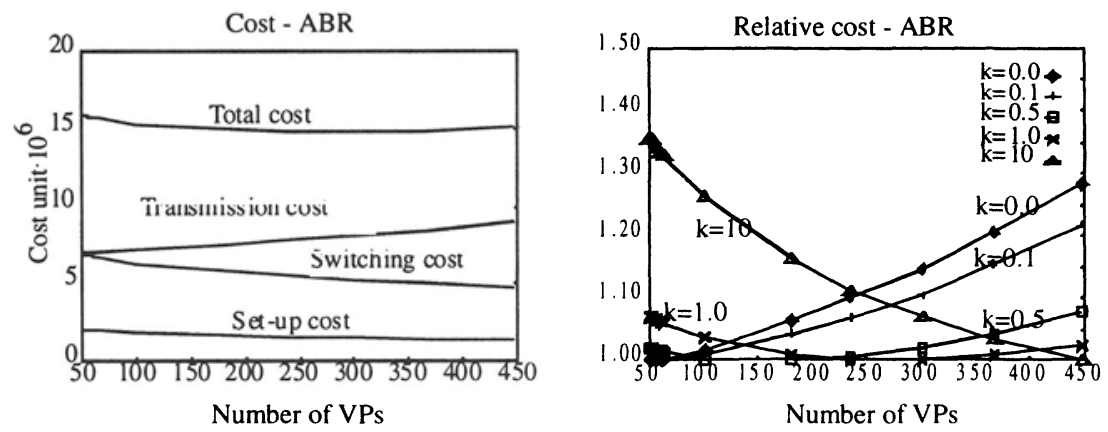

Figure 7. Results for ABR case: a) Cost as function of number of VPs, b) Total cost relative minimum total cost for selected values of set-up/switching weight factors

The minimum total cost for the ABR case with the weight factor, $k=1.0$, is obtained with 584 VPs established. Having 50 VPs gives a total cost that is. $7.3 \%$ more expensive than the better solution having 301 VPs, while the total cost is increased by $2.5 \%$ having 448 VPs. The number of VPs has less influence on the total network cost than in the reference case.

\subsection{Comparing the reference and ABR cases}

The network cost is considerable higher for a fully connected VP network in the reference case, since the equivalent bandwidth in case of SBR is increasing with a decreasing bandwidth per VP.
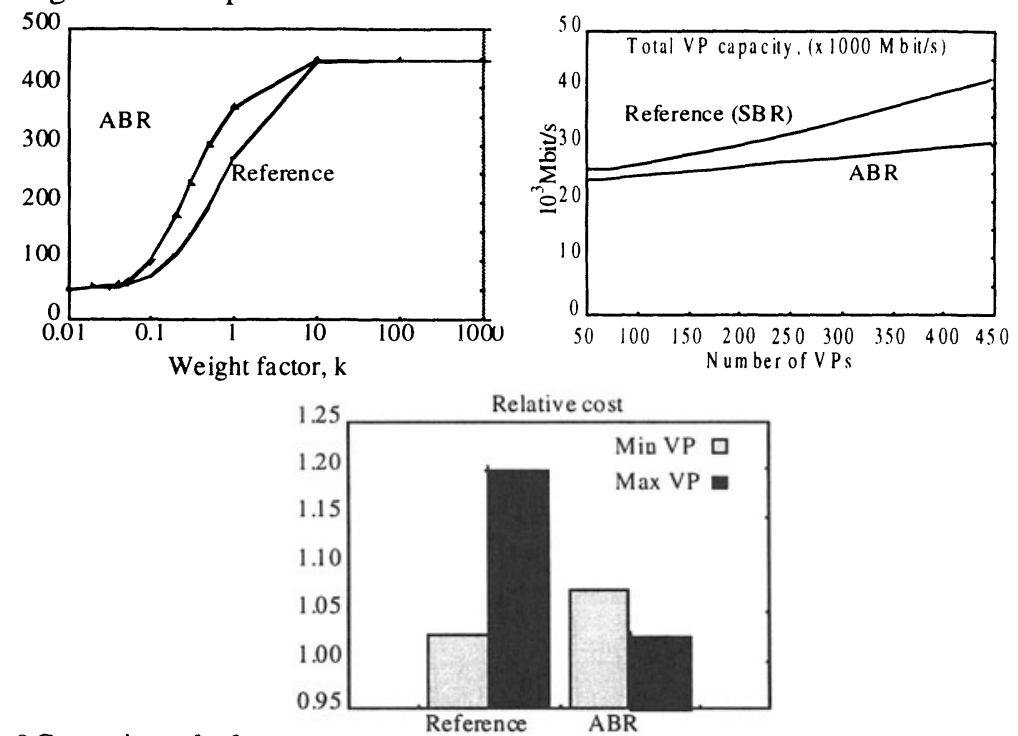

Figure 8 Comparison of reference case and ABR case: a) Number of VPs, b) Total cost relative minimum total, c) The total VP capacity in the network 
In the ABR case several VPs are established for lower values of the weight factor $k$ than in the reference case, as seen from Figure 8a). As seen from Figure 8b), the total capacity, when the number of VPs is 50 , is quite similar for the two cases, since the VPs have capacities huge enough to make the equivalent bandwidth close to the MCR/SCR. Cross connecting the VPs will make the capacity allocated to each VP lower and the equivalent bandwidth for the variable rate bitstreams will increase. Therefore, with a fully connected network, the reference case has a total capacity that is considerably higher than for the ABR case.

The relative network cost for the two cases is illustrated in Figure $8 c$ ). In the reference case the solution with the maximum number of VPs has a much higher network cost than the better solution with 146 VPs. The network cost with the maximum number of VPs is also much higher than the cost with the minimum number of VPs. In the ABR case, the relations of network costs are the opposite; the solution with a minimum number of VPs has a higher cost than the solution with the maximum number of VPs.

The various network solutions obtained, when varying the weight factor $k$, will provide different grade of service especially to the best-effort traffic. First, the capacity available in the reference case will be higher due to the dimensioning with equivalent bandwidth. This gives more "spare" capacity in the network solutions found for the reference case. Second, the number of VPs will reflect the degree of sharing of capacity.

\section{CONCLUSIONS AND FURTHER WORK}

As illustrated by the reference case and the ABR case, the characterisation of the offered traffic is essential for the network solution obtained when applying this algorithm. When the offered traffic is increased to a certain level for each $\mathrm{CoS}$, the influence on the equivalent bandwidth is decreased, and the equivalent bandwidth is tending towards the mean rate. The bandwidth required has no longer a strong dependency on the number of VPs, and finding the number of VPs in the better solution is no longer the main issue.

However, the network dimensioning tool for carrying out network design presented here, may be adapted to investigate other aims, e.g. by use of other cost, equivalent bandwidth approximations, buffer allocation schemes, and capacity reservation policies.

As already discussed in Chapter 2, VPs may be used for a number of reasons. In this paper the motivation has been to segregate bitstreams having different $\mathrm{CoS}$, to reduce the overall cost by cross-connecting VPs and to study the influence of application characterisation. When considering cross-connecting a VP, the switching and set-up costs in the intermediate node are compared to the cost of additional capacity necessary when splitting the VPs.

In broadband networks, the amount of offered best-effort traffic might be considerable, and the available bandwidth may be insufficient when the dimensioning is based on the MCR. The network dimensioning algorithm may be enhanced further to better incorporate best-effort type of traffic. The segregation scheme may also be changed to keep the best-effort traffic streams within VPs having a suitable traffic mixture. 


\section{References}

[1] Z. Dziong, J. Zhang, L. G. Mason: "Virtual Network Design - An Economic Approach", ITC, Lund, Sweden, 1996

[2] EURESCOM P616 Deliverable D2: Congestion Control in a VC Switched ATM Network. 1998.

[3] A. Farago, S. Blaabjerg, W. Holender, B. Stavenow, T. Henk, L. Ast, S. Szekely: "Enhancing ATM Network Performance by Optimizing the Virtual Network Configuration", IFIP Data Communication and their Performance, Istanbul, Turkey, 1995.

[4] ITU-T Rec. I-356: B-ISDN ATM layer cell transfer performance, 1999.

[5] ITU-T Rec. E.737: Dimensioning methods for B-ISDN, 1997.

[6] JAMES Project Deliverable D18: Report on use of ATM network planning guidelines. 1998.

[7] K. Lindberger: "Dimensioning and Design Methods for Integrated ATM Networks", In Proceedings of $14^{\text {th }}$ ITC, Vol. 1b,pg. 897-906, J. Labetouelle and J.W.Roberts(ed.), Antibes, France, June 1994

[8] J. Roberts, M. Mocci, J. Virtamo (ed.): Broadband Network Teletraffic - Final Report of Action COST 242, 1996.

[9] B. H. Ryu, H. Ohsaki, M. Murata, H. Miyahara: "Design Algorithm for Virtual Path based ATM networks", IEICE Trans. Communication, Vol. E79-B, No. 2, February 1996. 